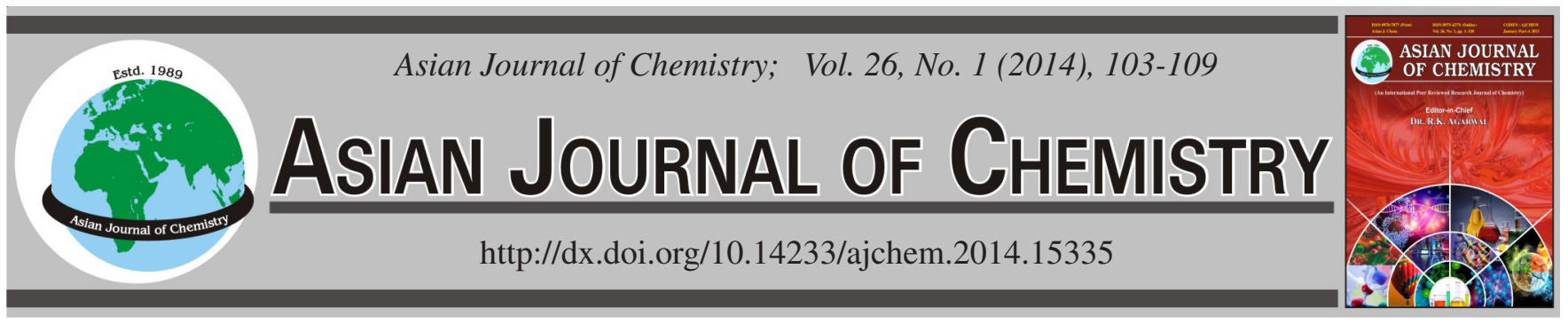

\title{
Analysis and Evaluation of Fatty Acid in Rattan Tea by Highly Selective and Sensitive HPLC- FLD-MS Method Coupled with Pre-Column Fluorescent Labeling
}

\author{
Baolei Fan ${ }^{1,2,3}$, Guang Chen ${ }^{1,3}$, Xiangming Hen $^{1,3}$ and Yourui Suo ${ }^{1, *}$
}

${ }^{1}$ Northwest Institute of Plateau Biology, Chinese Academy of Sciences, Xining, Qinghai Province, P.R. China
${ }^{2}$ Hubei University of Science and Technology, Xianning, Hubei Provice, P.R. China
${ }^{3}$ University of Chinese Academy of Science, Beijing, P.R. China

*Corresponding author: Fax: +86 370 97663482; Tel: +86971 6143857; E-mail: yrsuo@nwipb.cas.cn

Received: 18 February 2013;

Accepted: 9 May 2013;

Published online: 26 December 2013;

AJC-14482

\begin{abstract}
Rattan tea regarded as one of the most promising dietary supplements for the prevention and treatment of many chronic diseases has gained extensive attention. Free fatty acids play an important role in human body as essential nutrients. Contents of 31 free fatty acids in Rattan Tea were firstly investigated using toluene-4-sulfonic acid 2-(naphthalene-1-yl-phenyl-amino)-ethyl ester as pre-column fluorescence derivatization reagent by RE-HPLC coupled with an atmospheric chemical ionization-mass spectrometry source (APCI/MS). The results indicated that lower values of LOD $\left(0.52-1.53 \mathrm{ng} \mathrm{mL}^{-1}\right)$ provided the guarantee for trace analysis and evaluation of free fatty acids. The experimental recoveries were in the range of 88.9-103.6\%. Results of the present study indicated the highest free fatty acids content of

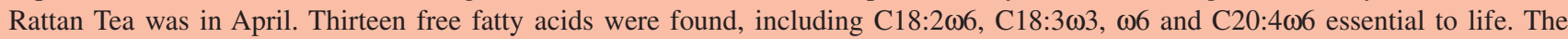
developed method exhibited powerful potential for the systematic detection of bioactive components of medicinal plants.
\end{abstract}

Keywords: Fatty acids, Rattan Tea, RE-HPLC-APCI/MS, Fluorescence detection, Pre-column derivatization.

ᄂ - - - - - - - - - - - - - - - - - - - - - - - - - - -

\section{INTRODUCTION}

Rattan tea, the tender stems and leaves of Ampelopsis grossedentata (Hand-Mazz) W.T. Wang, is mostly used for medicinal-edible purposes in Wuling mountain and Wuyi mountain regions ${ }^{1}$. It was reported that Rattan tea possessed a number of biological functions, including hypoglycemic, antithrombotic, antioxidant, antiinflammatory and antibacterial activities etc. ${ }^{2}$.

Due to its medicinal and nutritional uses, several phytochemicals of Rattan tea have been studied, including polysaccharide, sesquiterpene lactones and steroidal saponin ${ }^{3}$. Some analyses revealed high level of flavonoids in Rattan tea ${ }^{4,5}$. Being essential element of nutritional ingredient, free fatty acid (FFA) plays an independent role in herbal tea. One part of fatty acid is connected with compositions of herbal tea by enzymatic reaction $^{6}$; another part is unsaturated fatty acid (UFA), especially the omega- 3 and -6 series, is necessary for human health due to their effects on normal health and chronic diseases, such as the regulation of cholesterol levels, reduction of the risk of cardiovascular diseases, promotion of neuronal development and immune functions ${ }^{7}$. In this respect, sensitive and accurate analysis of FFAs in Rattan tea is of great importance. However, there is no report on the fatty acid profiles of Rattan tea, to the best of our knowledge.
Although FFA can be analyzed using gas chromatography (GC) or gas chromatography coupling with mass spectrometer $(\mathrm{GC}-\mathrm{MS})^{3}$, GC was widely used in the micro-scale analysis of fatty acids and long chain polyunsaturated fatty acid (PUFA) was unstable to be analyzed ${ }^{8}$. Compared with the aforementioned methods, high-performance liquid chromatography (HPLC) with ultraviolet (UV) or uorescence detection (FLD) as being highly sensitive, has been proven to be a more powerful analytical method ${ }^{9,10}$. But most fatty acids show neither natural UV absorption nor uorescence ${ }^{11}$. Analytes with labeling reagents, which produce intense fluorescence derivatives, can be more easily analyzed. Therefore, derivatization has been widely adopted, which can overcome some problems, such as tailing peaks and low detector sensitivity ${ }^{12}$.

The present paper is mainly concerned with the simultaneous separation and determination of 31 FFAs in combination with a selective and sensitive derivatization method using toluene-4-sulfonic acid 2-(naphthalene-1-yl-phenyl-amino)ethyl ester (TSNPE) as labeling reagent. The detection and identification were carried out, respectively by fluorescence and APCI/MS in positive-ion mode. At the same time, a comparative study on the FFA compositions of wild Rattan tea collected in April, July and September from Guizhou and Hunan was conducted. 


\section{EXPERIMENTAL}

Experiments of the HPLC system were performed on Agilent HP 1100 series consisting of an autosampler (model G1329A), a quaternary pump (model G1311A), a thermostated column compartment (model G1316A), a vacuum degasser (model G1322A) and a fluorescence detector (FLD) (model G1321A). The mass spectrometer 1100 Series LC/MSD TrapSL (ion trap) equipped with an APCI source (Bremen, Germany) was controlled by Esquire-liquid chromatography NT software (version 4.1). A reversed-phase Hypersil BDS C8 column ( $5 \mu \mathrm{m}, 200 \mathrm{~mm} \times 4.6 \mathrm{~mm}$ i.d.) by a gradient elution were used for derivatives separation. Fluorescence emission and excitation spectra were obtained at a F7000 fluorescence spectrophotometer (Hitachi, Japan).

Rattan tea of Guizhou was obtained from Fanjing mountain region (the peak of Wuling mountain" Man and Biosphere" protected areas of UNESCO), Guizhou province (China) 2011. Rattan tea of Hunan was obtained from Xiangxi Tujia and Miao Autonomous Prefecture, Hunan province (China) 2011. All the samples were collected in the same standard and processed in the same way by the Company of Miao medicine in Jiangkou, Guizhou. $500 \mathrm{~g}$ Rattan tea samples were dried and pulverized over 40 mesh sieve.

Toluene-4-sulfonic acid 2-(naphthalene-1-yl-phenylamino)ethyl ester (TSNPE) was synthesized in our laboratory. Standards of 31 FFAs were of chromatographic grade and purchased from Sigma Reagent Co. (USA). Spectroscopically pure acetonitrile (ACN) obtained from Yuwang Company, China. Water was puried on a Milli-Q system (Millipore, Bedford, MA, USA). N, potassium carbonate $\left(\mathrm{K}_{2} \mathrm{CO}_{3}\right), \mathrm{N}$ dimethylformamide (DMF), chloroform and pyridine were of analytical grade and purchased from Shanghai Chemical Reagent Co. (Shanghai, China). All other reagents used were also of analytical grade unless otherwise stated.

Extraction of free fatty acids from Rattan tea samples: To a $10 \mathrm{~mL}$ of round-bottom flask containing $10 \mathrm{~mL}$ chloroform, $2 \mathrm{~g}$ pulverized Rattan tea were added. The flask was immersed in an ultrasonic water bath for $0.5 \mathrm{~h}$. The extract was collected by filtration under vacuum. And $5 \mathrm{~mL}$ chloroform was added into the residue for further extraction. The two extracts were combined and $1.5 \mathrm{~mL}$ pyridine was added. Subsequently the extracts were immersed in an ultrasonic water bath for $20 \mathrm{~s}$ to convert it to the corresponding fatty acids organic salt. Finally, after being evaporated to dryness under a gentle stream of nitrogen, the extracts of Rattan tea were dissolved with $500 \mu \mathrm{L}$ DMF, stored in a refrigerator.

Preparation of standard solutions: Individual stock solutions of the FFAs $\left(1.0 \times 10^{-3} \mathrm{~mol} \mathrm{~L}^{-1}\right)$ were prepared in $\operatorname{ACN} / D M F(1: 1, v / v)$ and the concentration of $5.0 \times 10^{-5} \mathrm{~mol}$ $\mathrm{L}^{-1}$ were diluted with the same solvent composition. TSNPE solution $\left(1.0 \times 10^{-3} \mathrm{~mol} \mathrm{~L}^{-1}\right)$ was prepared by dissolving 4.16 $\mathrm{mg}$ TSNPE into $10 \mathrm{~mL}$ anhydrous acetonitrile. When not in use, all reagent solutions were stored at $4{ }^{\circ} \mathrm{C}$ in a refrigerator.

Derivatization: To a solution containing $20 \mu \mathrm{L}$ standard FFAs mixture (or extracted sample) in a vial, $200 \mu \mathrm{L}$ DMF, $100 \mu \mathrm{L}$ reagent solution and $10 \mathrm{mg} \mathrm{K}_{2} \mathrm{CO}_{3}$ were added, respectively. The vial was sealed and placed in water bather at $85^{\circ} \mathrm{C}$ for $35 \mathrm{~min}$ shaken in $5 \mathrm{~min}$ intervals. Then, the mixture was cooled to room temperature after the reaction finished. $400 \mu \mathrm{L} \mathrm{DMF}$ was added to dilute the derivatization solution. The diluted solution $(10 \mu \mathrm{L})$ was injected directly to the HPLC system. The derivatization procedure was shown in Fig. 1.

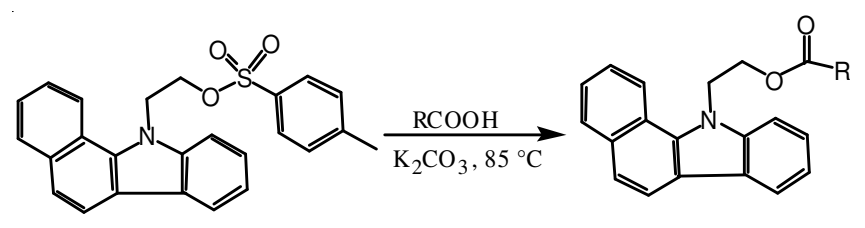

Fig. 1. Derivatization scheme of TSNPE with FFA

HPLC-MS conditions: HPLC separation of 31 FFAs derivatives was performed on a reversed-phase Hypersil BDS C8 column $(5 \mu \mathrm{m}, 200 \mathrm{~mm} \times 4.6 \mathrm{~mm}$ i.d. $)$ with a gradient elution (Table-1). The uorescence wavelengths were set to $\lambda_{\text {em }} 380 \mathrm{~nm}$ and $\lambda_{\mathrm{ex}} 279 \mathrm{~nm}$, respectively. Eluent A was a mixed solvent of ACN/DMF (1:1, v/v), B was water and C was acetonitrile $(100 \%)$. The injection volume was $10 \mu \mathrm{L}$. The column temperature was set at $30^{\circ} \mathrm{C}$ and the flow rate was

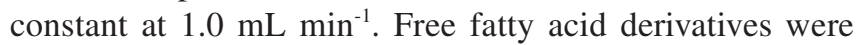
further identied by positive-ion APCI mass spectra. The APCI probe temperature, dry gas flow, dry gas temperature and nebulizer were set at $400{ }^{\circ} \mathrm{C}, 5.0 \mathrm{~L} \mathrm{~min}^{-1}, 350{ }^{\circ} \mathrm{C}$ and $60 \mathrm{psi}$, respectively.

\begin{tabular}{cccc}
\multicolumn{5}{c}{ TABLE-1 } \\
& GRADIENT ELUTION PROGRAM \\
\hline Time & A $(\%)$ & B $(\%)$ & C $(\%)$ \\
\hline 0 & 55 & 40 & 5 \\
15 & 70 & 20 & 10 \\
35 & 87 & 3 & 10 \\
50 & 88 & 2 & 10 \\
60 & 85 & 0 & 15 \\
\hline
\end{tabular}

\section{RESULTS AND DISCUSSION}

HPLC separation: The introduction of the 2-(11H-benzo[a]carbazol-11-yl) ethyl 4-methylbenzenesulfonate (TSNPE) molecule augmented the $n$-p conjugation and was favorable for the sensitive determination of free fatty acids. Although FFAs can be analyzed by both HPLC and GC, derivatization in GC analysis often showed instability and low sensitivity ${ }^{13,14}$. Considering the above factors, in this study, we adopted the liquid chromatography method.

To get a good evaluation of the fatty acids that may exist in Rattan tea, 31 fatty acids (unsaturated and saturated fatty acids) were chosen in this study. It was challenging to separate the 31 fatty acids in a HPLC column since unsaturated fatty acids often were co-eluted with saturated fatty acids containing one or two fewer carbon atoms. To achieve optimal separation of all the FFAs within the shortest time, several conditions were investigated to ensure satisfactory HPLC separation. A Hypersil BDS C8 column ( $5 \mu \mathrm{m}, 200 \mathrm{~mm} \times 4.6 \mathrm{~mm}$ i.d.) was selected in conjunction with a gradient elution as described in experimental section 2.7. With the chromatographic conditions described above, a satisfactory resolution for 31 fatty acid derivatives was obtained within $50 \mathrm{~min}$ and with sharp peaks (Fig. 2). 


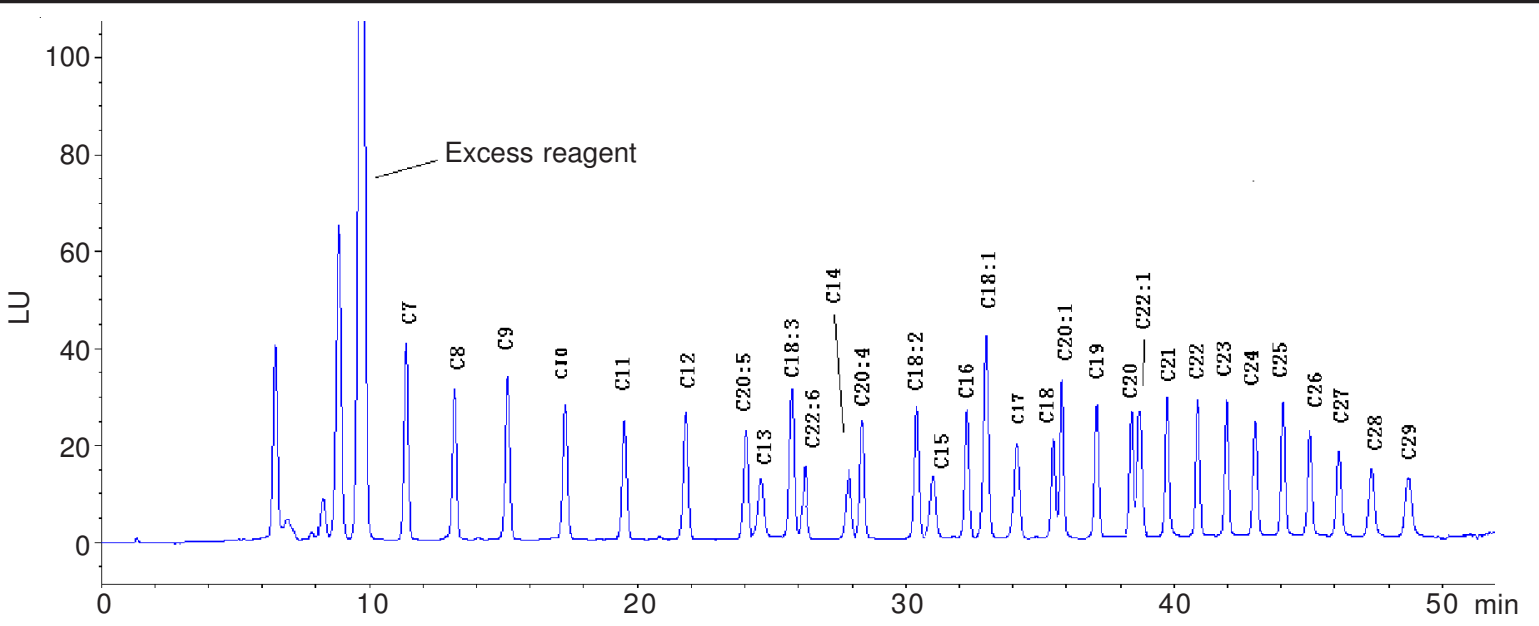

(a)

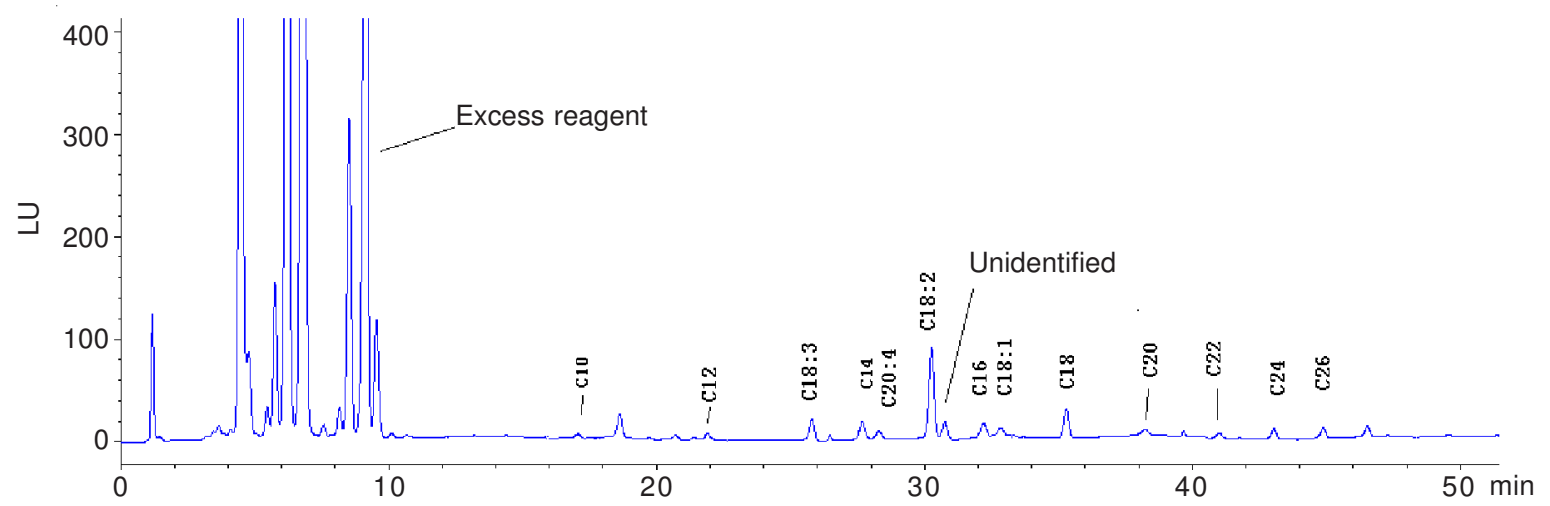

(b)

Fig. 2. (a) Chromatogram of a mixture of FFA standards. (b) Chromatogram of FFAs in Rattan Tea (April, Guizhou). Chromatographic conditions: Column temperature at $30^{\circ} \mathrm{C}$; excitation wavelength $\lambda_{\text {ex }} 279 \mathrm{~nm}$, emission wavelength $\lambda_{\text {em }} 380 \mathrm{~nm}$; Hypersil BDS C8 column $(5 \mu \mathrm{m} 200 \mathrm{~mm} \times 4.6 \mathrm{~mm}$ i.d.); flow rate $=1.0 \mathrm{~mL} \mathrm{~min}^{-1}$. Peaks: $\mathrm{C} 7$ (heptoic acid); $\mathrm{C} 8$ (octoic acid); $\mathrm{C}$ (pelargoic acid); $\mathrm{C} 10$ (decoic acid); $\mathrm{C} 11$ (undecanoic acid); $\mathrm{C} 12$ (dodecanoic acid); C13(tridecanoic acid); C20:5(5,8,11,14,17-eicosapentaenoic acid); C18:3(9,12,15- octadecatrienoic acid); C22:6(2,5,8,11,14,17-docosahexenoic acid); C14 (tetradecanoic acid); C20:4(6,9,12,15 -arachidonic acid); C18:2 (9,12-octadecadienoic acid); C15 (pentadecanoic acid); C16 (hexadecanoic acid); C18:1 (12-octadecenoic acid); C17(hep tadecanoic acid); C18(octadecanoic acid); C20:1 (11-eicosenoic acid); C19 (nonadecanoic acid); C20 (eicosoic acid); C22:1(12-docosenoic acid); C21(heneicosoic acid); C22 (docosanoic acid); C23(tri-cosanoic acid); C24(tetracosanoic acid); C25(pentacosanoic acid); C26(hexacosanoic acid); C27 (heptacosanoic acid); C28(octadecanoic acid); C29(nonacosanoic acid)

MS characterization of the TSNPE-FFAs: MS detection is very specific with respect to the type and shape of molecules, thus being able to provide important information on their fragmentation and to confirm their structure ${ }^{15}$. In order to correctly identify each FFA, the mass spectra of identification for TSNPE-FFAs were carried out.

With APCI/MS in positive ion detection mode, intense ion current signals for fatty acid derivatives should be attributed to the introduction of the weak basic nitrogen in corresponding TSNPE molecular core structure resulting in high ionization efficiency $^{16}$

MS characterization of the TSNPE -SFA: Because saturated fatty acid (SFA) is relatively stable, the positive-ion mass spectra corresponding to TSNPE-SFA was easily to be assigned; the collision-induced dissociation spectra for representative octadecanoic acid (OA, C18) derivative produced the specific fragment ions (Fig. 3). Owing to the existence of the nitrogen atom in the molecular core, TSNPE could easily transform to its isomer ${ }^{17}$. As expected, the TSNPE-C18 derivative produced an intense molecular ion peak at $\mathrm{m} / \mathrm{z} 527.8$ $[\mathrm{M}+\mathrm{H}]^{+}$. The MS/MS of molecular ions $\left(\mathrm{MS},[\mathrm{M}+\mathrm{H}]^{+}\right.$ion) produced intense and stable fragment ions at $\mathrm{m} / \mathrm{z}, 216.0,243.2$ and 312.0. The characteristic ions at $\mathrm{m} / \mathrm{z}, 216.0,243.2$ were common to all TSNPE-FFAs and $\mathrm{m} / \mathrm{z}, 312$ was the characteristic ion of TSNPE-C18. These ions were mainly produced by the cleavage of the molecular core within TSNPE. The specific fragment ions at $m / z 216.0$ and $m / z 312.0$ were from the cleavage of $\mathrm{N}-\mathrm{C}$ bond of the N-linked side chain, corresponding to the protonated molecular core of benzoacridine. The specific fragment ion at $m / z, 243.2$ was from the cleavage of C-O bond of the N-linked side chain, corresponding to the protonated vinyl-benzocarbazol and ethoxide-benzocarbazol moieties. The MS/MS analysis and corresponding cleavage mode stand for a representative TSNPE-C18 (Fig. 3a-b).

MS characterization of TSNPE-UFA: Fragment ions were particularly rich for unsaturated fatty acid (UFA) derivatives, which should be attributed to the cleavage of doublebonds in UFA molecules. The collision-induced dissociation spectra for the representative TSNPE-C18:2 16 (linoleic acid) produced the specific fragment ions at $\mathrm{m} / \mathrm{z}, 286.0, \mathrm{~m} / \mathrm{z}$ 149.9, $\mathrm{m} / \mathrm{z}$ 389.9, $\mathrm{m} / \mathrm{z} 467.6$ and an intense molecular ion peak at $\mathrm{m} / \mathrm{z}$ $523.2[\mathrm{M}+\mathrm{H}]^{+}$(Fig. 4). Fragment ions at $\mathrm{m} / \mathrm{z}, 149.9$ and $\mathrm{m} / z$ 467.6 were due to the cleavage of $\beta$ carbon-carbon bond (cleavage of allylic) ${ }^{18}$. Fragment ion at $m / z 286.0$ was produced by 

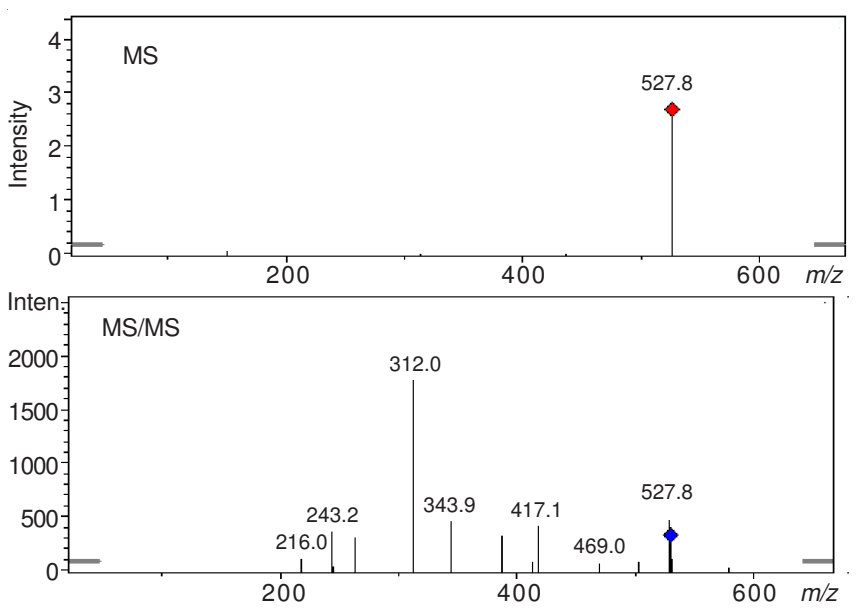

(a)

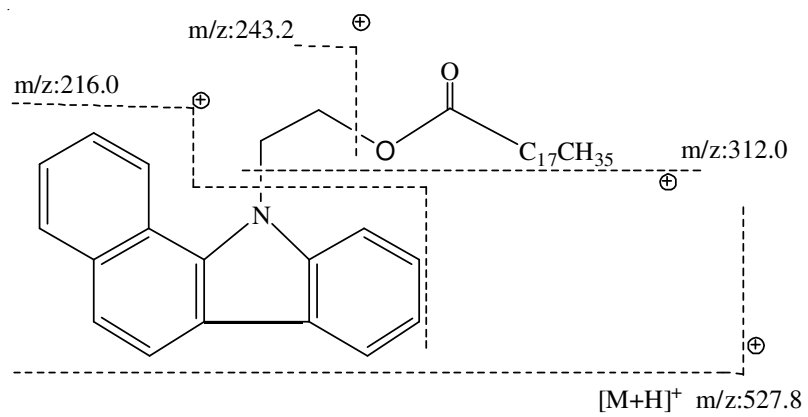

(b)

Fig. 3. Scheme of MS spectra of representative TSNPE-labeled C18 derivative: a molecular ion MS; MS/MS. Scanning range from 100 to $1000 \mathrm{amu}$ under APCI positive-ion mode. $\mathrm{b}$ MS cleavage mode of representative octadecanoic acid $(\mathrm{OA}, \mathrm{C} 18)$ derivative

the cleavage of $\alpha$ carbon-carbon and loss of the larger alkyl ${ }^{19}$. The specific fragment ion at $\mathrm{m} / \mathrm{z} 389.9$ was produced by means of McLafferty rearrangement to the double bond on the terminal side of the carbon chain and the conjugated double bonds come into being ${ }^{20}$. The MS/MS analysis and corresponding cleavage mode stood for a representative TSNPE-C18:2 $\omega 6$ showing in Fig. 4a-b. $[\mathrm{M}+\mathrm{H}]^{+}$Data for all the fatty acid derivatives were shown in Table-2.

Identification by HPLC-MS: Identification of compounds by HPLC-MS analysis was carried out by comparing retention times and mass spectra of the unknown peaks to those of the standards. Fig. 2 showed that although there was a peak with its retention time approximate to $\mathrm{C} 15$, Mass identification demonstrated no absolutely mass spectrometry information associated with TSNPE-C15. Thus, the coincident peak time for both this impurity and TSNPE-C15 fatty acids was probably caused by the small molecule acid marked with more than one carbazole rings, which is need to be identified further. The application of MS detection can be extremely useful for the study of more complex matrices, such as other herb extracts.

Validation of the method: Method validation requires an evaluation of the linearity, LOD, LOQ, precision and accuracy. The linear regression parameters were shown in Table-2. All 31 FFAs were found to show good linear responses with correlation coefficients of $>0.9992$, indicating excellent linearity of the analytes. The LOD calculated at a signal-tonoise ratio $(\mathrm{S} / \mathrm{N})$ of 3 were in the range of $0.52-1.53 \mathrm{ng} \mathrm{mL}^{-1}$,
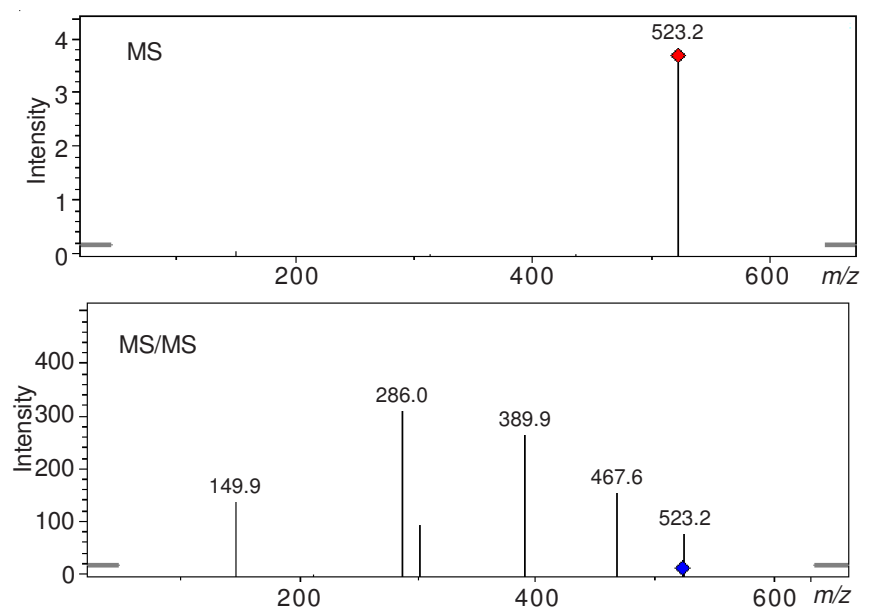

(a)
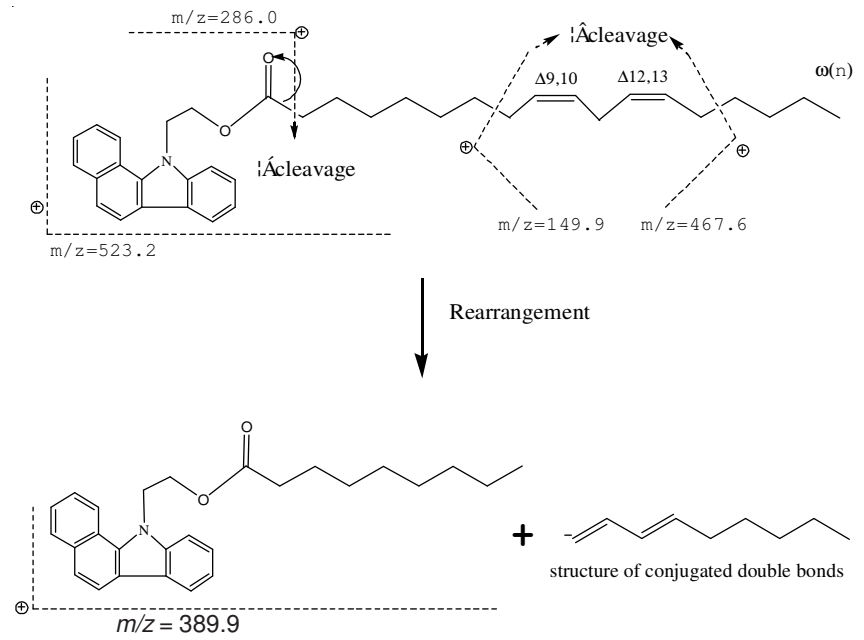

(b)

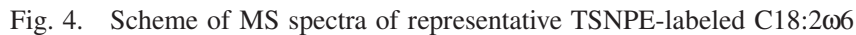
derivative: a molecular ion MS; MS/MS. Scanning range from 100 to $1000 \mathrm{amu}$ under APCI positive-ion mode. (b) MS cleavage mode

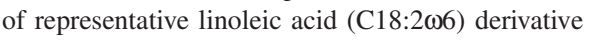

while the LOQ at a signal-to-noise ratio $(\mathrm{S} / \mathrm{N})$ of 10 were in the range of 1.32-4.09 $\mathrm{ng} \mathrm{mL}^{-1}$. The relative standard deviations (RSDs) of the peak areas and retention times for TSNPE derivatives varied from 0.18 to 0.60 and $0.02-0.25 \%$, respectively. The experimental recoveries were in the range of 88.9 to $103.6 \%(n=6)($ Table-2). All the data indicated that the linearity, detection limits, precision and recovery were satisfactory.

Free fatty acids analysis in Rattan tea: The developed method was successfully applied to analyze FFAs of Rattan tea collected in April, July and September from Guizhou and Hunan, respectively. The saturated and unsaturated fatty acids could be simultaneously separated in 50 min with a good baseline resolution and qualitatively identified by mass spectrometric analysis. The representative chromatograms were shown in Fig. 2 and the compositions and contents of fatty acids were given in Table- 3 .

Results of the study indicate that the major fatty acids

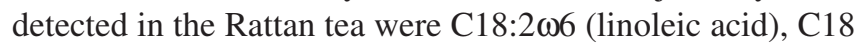

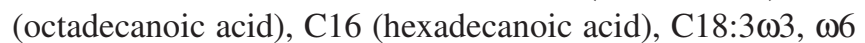

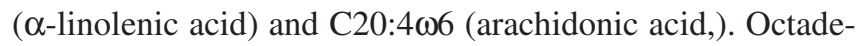
canoic acid (44.96-23.64 $\mu \mathrm{g} \mathrm{g}^{-1}$ ) was identified as the primary 
SFA, while linoleic acid (120.86-60.67 $\left.\mu \mathrm{g} \mathrm{g}^{-1}\right)$ was the primary unsaturated fatty acid. Meanwhile, the same types of FFAs in Rattan tea between Guizhou and Hunan showed geographical differentiation among populations, whereas FFA compositions had uniform consistency at the species level, revealing stable genetic diversity of this species ${ }^{21}$.
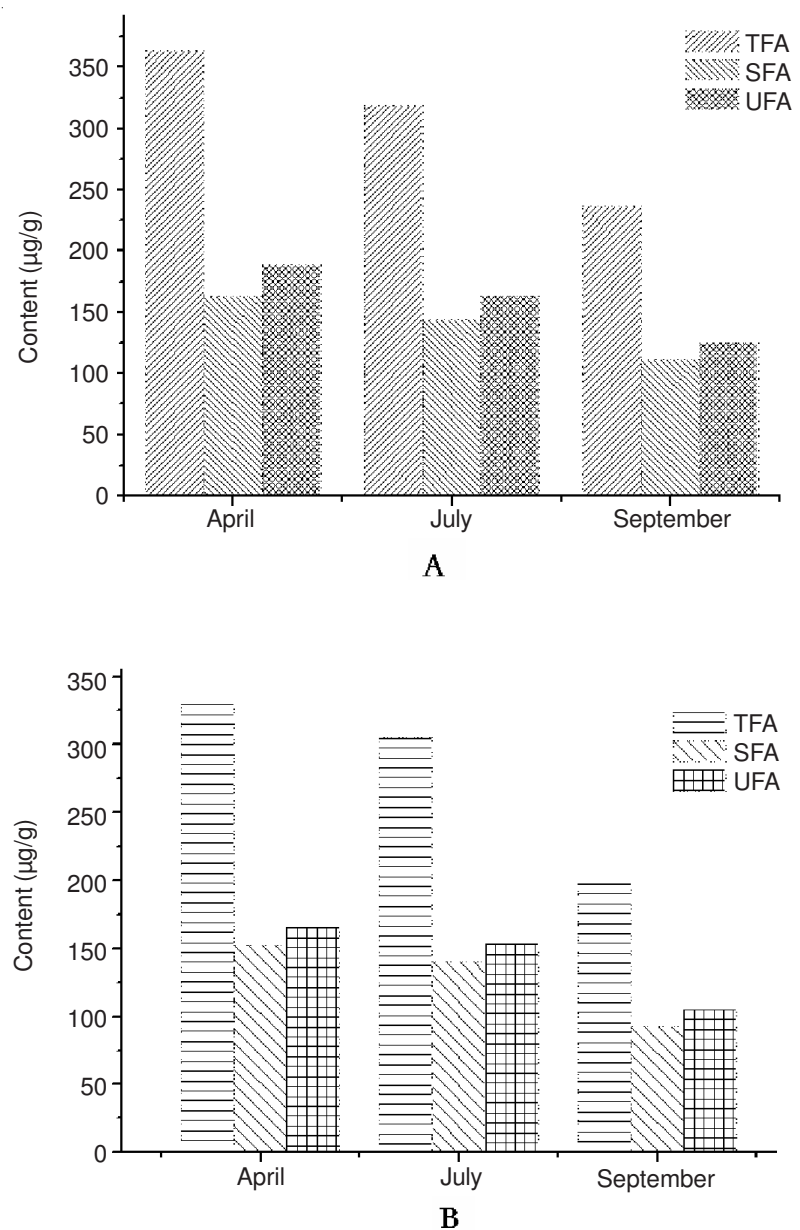

Fig. 5. Content and composition of fatty acids in April, July and September. A: The Rattan tea from Guizhou; B: The Rattan tea from Hunan

From the Table-3 and Figs. 5 \& 6, we also can see (1) Rattan tea under the same collecting and processing standards were as follows: April, July and September, from high to low concentration. Maybe it is related to climate. When the temperatures from June to September are high, the growth of Rattan tea is fast, but the accumulation of material in the plant decrease $\mathrm{e}^{22}$. (2) The content of Rattan tea in Guizhou was higher than that in Hunan, which was probably connected with the climatic conditions and certain geographical environment of the two places. For example, the elevation of the former is higher than the latter ${ }^{23}$. (3) The UFA contents of Rattan tea were generally higher than SFA, in April, July and September of the two places, respectively.

Significance of free fatty acids in Rattan tea: The consumption of fat in the modern age is remarkable compared with the past and major causes of death such as degenerative cardiovascular diseases and cancer are linked to aspects of dietary fat intake ${ }^{24,25}$. This led to the recommendation that dietary intake of UFA, especially poly unsaturated fatty acid

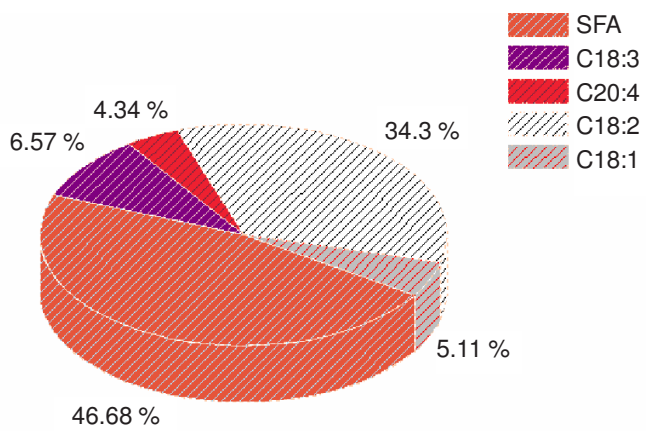

A

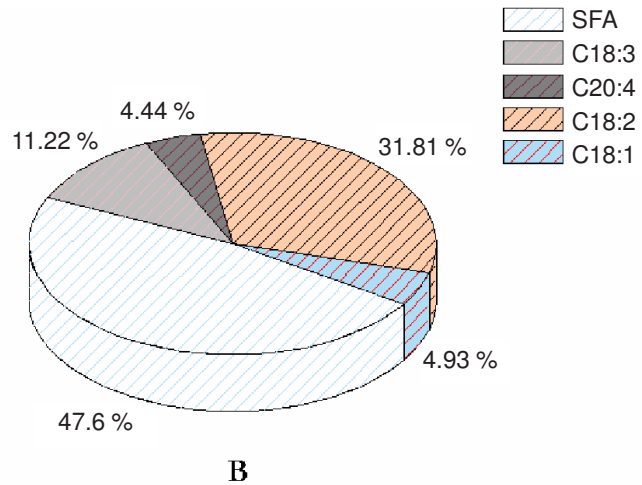

Fig. 6. Composition of average fatty acids of Rattan tea in April, July, September A: The Rattan tea from Guizhou; B: The Rattan tea from Hunan

$(\text { PUFA })^{26}$. In our research, the results revealed that poly unsaturated fatty acids (PUFA) in Rattan tea contain linoleic acid, $\alpha$-linolenic acid and arachidonic acid. The values of the PUFA/SFA ratio in two places studied ranged from 1.03 to 1.00 which were all higher than the minimum value for the PUFA/SFA ratio recommended by HMSO (the recommended minimum value is 0.45$)^{27}$. Therefore, Rattan tea in Guizhou and Hunan are all healthy and nutritious in terms of their values of PUFA/SFA ratio.

Among UFA, the highest level in Rattan tea of Guizhou

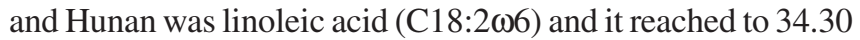
and $31.81 \%$, respectively. Linoleic acid is known to be one of the essential fatty acids, which must be obtained from food and can be synthesized little by animal self ${ }^{28}$. But in body of animals, the linoleic acid can be converted into arachidonic $\operatorname{acid}^{26}$ which was an important precursor for prostaglandin and thromboxane. Meanwhile, the Rattan tea have higher contents

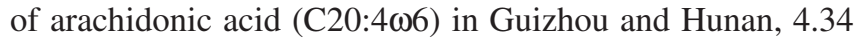
and $4.44 \%$, respectively.

Conjugated linoleic acid (CLA) is second level of UFA in Rattan tea, which is a long chain $\omega 3$ PUFA and must be obtained from the diet ${ }^{24,29}$. If we attempt to maintain or enhance our present-day health, one must insure to intake enough $\omega 3$ PUFA, which can prevent human from coronary artery disease $\mathrm{else}^{28,30}$. This study indicated the level of CLA $(\mathrm{C} 20: 3 \omega 3)$ in Rattan tea from Guizhou $(9.57 \%)$ was lower that of Hunan $(11.22 \%)$ counterparts.

In addition, FFA is the key role in the processing of aroma formation. As linoleic acid, $\alpha$-linolenic acid can form cis-3- 
$[\mathrm{M}+\mathrm{H}]^{+}$DATA, LINEAR REGRESSION EQUATIONS, CORRELATION COEFFICIENTS, LOD, LOQ, REPRODUCIBILITY OF RETENTION TIME AND PEAK AREA AND RECOVERY

\begin{tabular}{|c|c|c|c|c|c|c|c|c|}
\hline \multirow{2}{*}{ FFAs } & \multirow{2}{*}{$\begin{array}{c}\mathrm{m} / \mathrm{z}[\mathrm{M}+ \\
\mathrm{H}]^{+}\end{array}$} & \multirow{2}{*}{ Regression equation* } & \multirow{2}{*}{$\begin{array}{l}\text { Correlation } \\
\text { coefficient }\end{array}$} & \multirow{2}{*}{$\begin{array}{c}\text { LOD } \\
\left(\mathrm{ng} \mathrm{mL}^{-1}\right)\end{array}$} & \multirow{2}{*}{$\begin{array}{c}\text { LOQ } \\
\left(\mathrm{ng} \mathrm{mL}^{-1}\right)\end{array}$} & \multicolumn{2}{|c|}{$\operatorname{RSD} \%(n=6)$} & \multirow{2}{*}{$\begin{array}{c}\text { Recovery } \\
(\%)(\mathrm{n}=6)\end{array}$} \\
\hline & & & & & & Retention time & Peak area & \\
\hline $\mathrm{C7}$ & 374.1 & $Y=52.13 X+16.82$ & 0.9995 & 1.07 & 3.23 & 0.13 & 0.60 & 92.2 \\
\hline $\mathrm{C} 8$ & 388.3 & $Y=61.05 X+7.10$ & 0.9998 & 1.19 & 3.06 & 0.11 & 0.58 & 89.8 \\
\hline $\mathrm{C} 9$ & 402.3 & $Y=37.83 X+12.18$ & 0.9999 & 0.68 & 1.35 & 0.10 & 0.59 & 96.6 \\
\hline $\mathrm{C} 10$ & 416.3 & $Y=31.92 X+43.69$ & 0.9995 & 0.54 & 1.32 & 0.07 & 0.46 & 98.1. \\
\hline $\mathrm{C} 11$ & 430.1 & $Y=42.89 X+21.93$ & 0.9994 & 0.59 & 1.86. & 0.08 & 0.50 & 92.6 \\
\hline $\mathrm{C} 12$ & 444.3 & $Y=32.39 X+22.29$ & 0.9998 & 0.78 & 2.08 & 0.06 & 0.48 & 96.2 \\
\hline $\mathrm{C} 20: 5$ & 546.1 & $Y=61.23 X+62.59$ & 0.9995 & 1.53 & 4.09 & 0.06 & 0.38 & 96.1 \\
\hline C13 & 458.3 & $Y=56.68 X+33.24$ & 0.9992 & 1.22 & 3.09 & 0.06 & 0.22 & 95.5 \\
\hline $\mathrm{C} 18: 3$ & 521.9 & $Y=68.82 X+73.06$ & 0.9993 & 0.72 & 2.79 & 0.06 & 0.55 & 93.8 \\
\hline C22:6 & 572.1 & $Y=71.95 X+42.12$ & 0.9996 & 1.23 & 3.69 & 0.06 & 0.30 & 97.5 \\
\hline $\mathrm{C} 14$ & 472.3 & $Y=36.72 X-42.22$ & 0.9992 & 0.92 & 3.08 & 0.04 & 0.27 & 92.6 \\
\hline C20:4 & 547.9 & $Y=83.72 X-12.56$ & 0.9996 & 1.01 & 3.80 & 0.07 & 0.33 & 98.3 \\
\hline C18:2 & 523.9 & $Y=59.56 X+25.48$ & 0.9996 & 1.16 & 3.02 & 0.04 & 0.27 & 102.6 \\
\hline C15 & 486.3 & $Y=32.87 X+50.68$ & 0.9992 & 0.68 & 2.62 & 0.04 & 0.19 & 95.8 \\
\hline C18:1 & 525.8 & $\mathrm{Y}=61.09 \mathrm{X}+33.22$ & 0.9996 & 1.28 & 3.90 & 0.03 & 0.24 & 103.6 \\
\hline $\mathrm{C} 17$ & 514.4 & $Y=32.85 X+23.32$ & 0.9994 & 1.19 & 3.60 & 0.03 & 0.19 & 92.2 \\
\hline $\mathrm{C} 18$ & 527.8 & $Y=40.97 X+62.52$ & 0.9996 & 1.08 & 3.09 & 0.02 & 0.18 & 97.8 \\
\hline C20:1 & 553.9 & $Y=71.16 X+52.23$ & 0.9995 & 1.20 & 3.22 & 0.04 & 0.31 & 100.9 \\
\hline C19 & 542.4 & $Y=62.52 X+32.90$ & 0.9998 & 1.01 & 3.23 & 0.02 & 0.29 & 97.9 \\
\hline $\mathrm{C} 20$ & 556.3 & $Y=29.34 X+40.35$ & 0.9996 & 0.95 & 2.69 & 0.04 & 0.30 & 96.1 \\
\hline C22:1 & 582.6 & $Y=72.67 X+53.62$ & 0.9996 & 1.32 & 2.90 & 0.09 & 0.32 & 98.4 \\
\hline $\mathrm{C} 21$ & 570.3 & $Y=35.95 X+27.90$ & 0.9998 & 1.22 & 3.77 & 0.07 & 0.23 & 96.4 \\
\hline $\mathrm{C} 22$ & 584.1 & $Y=43.64 X+32.65$ & 0.9996 & 1.02 & 3.76 & 0.09 & 0.25 & 88.9 \\
\hline $\mathrm{C} 23$ & 598.4 & $Y=38.21 X+22.16$ & 0.9996 & 1.12 & 3.50 & 0.12 & 0.38 & 95.2 \\
\hline $\mathrm{C} 24$ & 612.3 & $Y=56.23 X-69.98$ & 0.9998 & 0.52 & 1.66 & 0.19 & 0.32 & 97.6 \\
\hline $\mathrm{C} 25$ & 626.5 & $Y=41.62 X+32.06$ & 0.9999 & 1.09 & 3.22 & 0.25 & 0.33 & 101.6 \\
\hline $\mathrm{C} 26$ & 640.3 & $Y=29.29 X+34.23$ & 0.9997 & 1.03 & 3.10 & 0.23 & 0.36 & 91.9 \\
\hline $\mathrm{C} 27$ & 654.6 & $Y=42.80 X+42.40$ & 0.9996 & 1.13 & 3.58 & 0.25 & 0.23 & 96.6 \\
\hline
\end{tabular}

TABLE-3

COMPOSITION AND CONTENT OF FFAS FROM EXTRACTED RATTAN TEA $\left(\mathrm{n}=6, \mu \mathrm{g} \mathrm{g}^{-1}\right)$

\begin{tabular}{|c|c|c|c|c|c|c|}
\hline \multirow{2}{*}{ FFA } & \multicolumn{3}{|c|}{ Guizhou } & \multicolumn{3}{|c|}{ Hunan } \\
\hline & April & July & September & April & July & September \\
\hline $\mathrm{C} 10$ & $13.26 \pm 0.17$ & $12.56 \pm 0.12$ & $9.86 \pm 0.21$ & $12.21 \pm 0.23$ & $12.26 \pm 0.22$ & $9.86 \pm 0.07$ \\
\hline $\mathrm{C} 12$ & $9.62 \pm 0.21$ & $8.48 \pm 0.82$ & $7.78 \pm 0.07$ & $11.21 \pm 0.08$ & $8.82 \pm 0.07$ & $8.86 \pm 0.11$ \\
\hline $\mathrm{C} 14$ & $17.68 \pm 1.05$ & $16.75 \pm 1.17$ & $17.58 \pm 1.12$ & $9.97 \pm 0.12$ & $10.79 \pm 0.65$ & $8.56 \pm 0.09$ \\
\hline $\mathrm{C} 16$ & $26.56 \pm 0.56$ & $23.24 \pm 0.06$ & $21.15 \pm 0.09$ & $29.65 \pm 0.19$ & $25.21 \pm 0.20$ & $17.35 \pm 0.05$ \\
\hline $\mathrm{C} 18$ & $44.96 \pm 1.77$ & $39.79 \pm 0.51$ & $28.43 \pm 1.23$ & $32.95 \pm 0.78$ & $28.57 \pm 1.09$ & $23.64 \pm 0.77$ \\
\hline $\mathrm{C} 20$ & $9.73 \pm 0.12$ & $7.21 \pm 0.12$ & $6.49 \pm 0.06$ & $8.83 \pm 0.23$ & $5.32 \pm 0.06$ & $4.32 \pm 0.07$ \\
\hline $\mathrm{C} 22$ & $10.02 \pm 0.23$ & $8.89 \pm 0.03$ & $8.75 \pm 0.11$ & $10.07 \pm 0.22$ & $9.98 \pm 0.15$ & $7.96 \pm 0.17$ \\
\hline $\mathrm{C} 24$ & $14.48 \pm 0.39$ & $12.66 \pm 0.24$ & $9.23 \pm 0.14$ & $21.26 \pm 1.12$ & $20.62 \pm 0.56$ & $9.28 \pm 0.67$ \\
\hline $\mathrm{C} 26$ & $15.07 \pm 0.08$ & $14.22 \pm 0.03$ & $14.33 \pm 1.07$ & $15.76 \pm 0.50$ & $11.11 \pm 0.34$ & $10.33 \pm 0.31$ \\
\hline$\Sigma \mathrm{SFA}$ & 161.37 & 143.80 & 123.60 & 151.91 & 132.68 & 100.16 \\
\hline $\mathrm{C} 18: 3 \omega 3, \omega 6$ & $35.05 \pm 1.07$ & $30.77 \pm 0.32$ & $19.86 \pm 0.23$ & $35.07 \pm 1.21$ & $29.77 \pm 1.01$ & $25.86 \pm 1.12$ \\
\hline $\mathrm{C} 20: 4 \omega 6$ & $14.89 \pm 0.05$ & $12.11 \pm 0.04$ & $11.84 \pm 0.26$ & $13.78 \pm 0.15$ & $11.16 \pm 0.49$ & $10.91 \pm 0.35$ \\
\hline 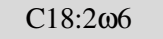 & $120.86 \pm 3.26$ & $105.44 \pm 2.70$ & $80.78 \pm 1.78$ & $101.25 \pm 3.09$ & $95.22 \pm 3.98$ & $60.67 \pm 2.13$ \\
\hline$\Sigma$ UFA & 188.62 & 163.38 & 125.37 & 165.21 & 153.10 & 105.20 \\
\hline$\Sigma \mathrm{TFA}^{*}$ & 349.99 & 307.18 & 248.97 & 317.12 & 285.78 & 205.36 \\
\hline
\end{tabular}

hexenal by breakage of a double bond between $\mathrm{C} 12-\mathrm{C} 15$ and the latter can be reverted into Aoba alcohol and Aoba aldehyde, which were the precursor of various aroma ${ }^{6}$. On the other hand, the quality of Rattan tea deteriorated easily by oxidation of UFA during storage.

\section{Conclusion}

In this study, using TSNPE as labeling reagent with fluorescence detection coupled with MS, quantitative determination and qualitative identification of 31 FFAs were simultaneously performed in wild Rattan tea. Under the chromato- 
graphic conditions, 31 FFAs were successfully separated in 50 min with satisfactory results. The developed method showed good correlation and high sensitivity, which could be used for the rapid determination and evaluation of FFAs in more diverse medicinal plants.

There were 13 FFAs found in Rattan tea, including linoleic acid, arachidonic acid and $\alpha$-linolenic acid that are essential to life. Results of the present study indicated the Rattan tea contained highest FFAs in April among the three months from Guizhou and Hunan, respectively. The research results will provide theory and reference for product research and development of wild Rattan tea.

\section{ACKNOWLEDGEMENTS}

This work was supported by Western Light Project of The Chinese Academy of Science (2010-2013) and the Plan Projects of Science and Technology of Tongren City, Guizhou (2012).

\section{REFERENCES}

1. J. Gao, B. Liu, Z. Ning, R. Zhao, A. Zhang and Q. Wu, J. Food Biochem., 33, 808 (2009).

2. Q. Zheng, L. Xu, L. Zhu, J. Chen, F. Liu, D. Chen, K. Xu and M. Chen, Bull. Mater. Sci., 33, 273 (2010).

3. Z. Luo, X. Fu and M. Wu, Food Sci., 26, 513 (2005).

4. N. Volpi and G. Bergonzini, J. Pharm. Biomed. Anal., 42, 354 (2006).

5. Q. Du, P. Chen, G. Jerz and P. Winterhalter, J. Chromatogr. A, 1040, 147 (2004).

6. G.W. Sanderson and H.N. Grahamm, J. Agric. Food Chem., 21, 576 (1973).

7. X. Kou and N. Chen, Food Sci. Human Wellness, 1, 14 (2012).

8. S. Thurnhofer and W. Vetter, J. Agric. Food Chem., 53, 8896 (2005).
9. H. Yang, Y. Ge, Y. Sun, D. Liu, X. Ye and D. Wu, Food Chem., 128, 1128 (2011).

10. C. Proestos, D. Sereli and M. Komaitis, Food Chem., 95, 44 (2006).

11. M. Reich, C. Hannig, A. Al-Ahmad, R. Bolek and K. Kümmerer, J. Lipid Res., 53, 2226 (2012).

12. Z. Sun, J. You, C. Song and L. Xia, Talanta, 85, 1088 (2011).

13. K. Takahashi and N. Goto-Yamamoto, J. Chromatogr. A, 1218, 7850 (2011).

14. M. Schreiner, J. Chromatogr. A, 1095, 126 (2005),

15. M. Pelillo, B. Biguzzi, A. Bendini, T. Gallina Toschi, M. Vanzini and G. Lercker, Food Chem., 78, 369 (2002).

16. J. You, L. Liu, W. Zhao, X. Zhao, Y. Suo, H. Wang and Y. Li, Anal. Bioanal. Chem., 387, 2705 (2007).

17. J. You, Y. Fu, Z. Sun and Y. Suo, Anal. Bioanal. Chem., 396, 2657 (2010).

18. K.B. Tomer, F.W. Crow and M.L. Gross, J. Am. Chem. Soc., 105, 5487 (1983).

19. L. Hejazi, D. Ebrahimi, M. Guilhaus and D.B. Hibbert, J. Am. Soc. Mass Spectrom., 20, 1272 (2009).

20. T. Nevigato, M. Masci, E. Orban, G. Di Lena, I. Casini and R. Caproni, Lipids, 47, 741 (2012).

21. J. Sun, F. Li, W. Xu, G. Zhou, J. You and G. Chen, Chromatographia, 70, 1627 (2009).

22. G.O. Guler, B. Kiztanir, A. Aktumsek, O.B. Citil and H. Ozparlak, Food Chem., 108, 689 (2008).

23. J. Xu, B. Yan, Y. Teng, G. Lou and Z. Lu, J. Food Compos. Anal., 23, 401 (2010).

24. M. Sugano and F. Hirahara, Am. J. Clin. Nutr., 71, 189S (2000).

25. A. Ohta, M.C. Mayo, N. Kramer and W.E.M. Lands, Lipids, 25, 742 (1990).

26. J. Beare-Rogers, A. Dieffenbacher and J. Holm, Pure Appl. Chem., 73, 685 (2001).

27. K. Justi, C. Hayashi, J. Visentainer, N. De Souza and M. Matsushita, Food Chem., 80, 489 (2003).

28. H. Poirier, I. Niot, L. Clement, M. Guerre-Millo and P. Besnard, Biochimie, 87, 73 (2005).

29. T.A.B. Sanders, Am. J. Clin. Nutr., 71, 176s (2000).

30. A. Simopoulos, Poult. Sci., 79, 961 (2000). 